\title{
Homoclinic Solutions for a Class of Second Order Nonautonomous Singular Hamiltonian Systems
}

\author{
Ziheng Zhang, ${ }^{1}$ Fang-Fang Liao, ${ }^{2}$ and Patricia J. Y. Wong ${ }^{3}$ \\ ${ }^{1}$ Department of Mathematics, Tianjin Polytechnic University, Tianjin 300387, China \\ ${ }^{2}$ Department of Mathematics, College of Science, Hohai University, Nanjing 210098, China \\ ${ }^{3}$ School of ELectrical and Electronic Engineering, Nanyang Technological University, 50 Nanyang Avenue, Singapore 639798
}

Correspondence should be addressed to Ziheng Zhang; zhzh@mail.bnu.edu.cn

Received 3 December 2013; Accepted 10 January 2014; Published 19 February 2014

Academic Editor: Jifeng Chu

Copyright (C) 2014 Ziheng Zhang et al. This is an open access article distributed under the Creative Commons Attribution License, which permits unrestricted use, distribution, and reproduction in any medium, provided the original work is properly cited.

\begin{abstract}
We are concerned with the existence of homoclinic solutions for the following second order nonautonomous singular Hamiltonian systems $\ddot{u}+a(t) W_{u}(u)=0$, (HS) where $-\infty<t<+\infty, u=\left(u_{1}, u_{2}, \ldots, u_{N}\right) \in \mathbb{R}^{N}(N \geq 3), a: \mathbb{R} \rightarrow \mathbb{R}$ is a continuous bounded function, and the potential $W: \mathbb{R}^{N} \backslash\{\xi\} \rightarrow \mathbb{R}$ has a singularity at $0 \neq \xi \in \mathbb{R}^{N}$, and $W_{u}(u)$ is the gradient of $W$ at $u$. The novelty of this paper is that, for the case that $N \geq 3$ and (HS) is nonautonomous (neither periodic nor almost periodic), we show that (HS) possesses at least one nontrivial homoclinic solution. Our main hypotheses are the strong force condition of Gordon and the uniqueness of a global maximum of $W$. Different from the cases that (HS) is autonomous $(a(t) \equiv 1)$ or (HS) is periodic or almost periodic, as far as we know, this is the first result concerning the case that (HS) is nonautonomous and $N \geq 3$. Besides the usual conditions on $W$, we need the assumption that $a^{\prime}(t)<0$ for all $t \in \mathbb{R}$ to guarantee the existence of homoclinic solution. Recent results in the literature are generalized and significantly improved.
\end{abstract}

\section{Introduction}

As is known to all, the search for periodic as well as homoclinic and heteroclinic solutions of Hamiltonian systems has a long and rich history. In present paper, we particularly focus our attention on the existence of homoclinic solutions of second order nonautonomous singular Hamiltonian systems. For the results on the literature of periodic solutions for such singular systems, we refer the reader to the book [1] of Ambreosetti and Zelati.

Second order Hamiltonian systems are systems of the following form:

$$
\ddot{u}+V_{u}(t, u)=0,
$$

where $t \in \mathbb{R}$ and $u \in \mathbb{R}^{N}$. Roughly speaking, they are the Euler-Lagrange equations of the functional

$$
I(u)=\int L(t, u, \dot{u}) d t
$$

where the integration is taken over a finite interval $[0, T]$ or all real $\mathbb{R}$ and the Lagrangian has the form

$$
L(t, u, \dot{u})=\frac{1}{2}|\dot{u}|^{2}-V(t, u) .
$$

Clearly, when the potential $V(t, u)$ is $T$-periodic in $t$, it is natural to look for $T$-periodic solutions of (HS) as critical points of the functional $I(u)$ over a suitable space of $T$-periodic functions. Also, in such a case, one can look for homoclinic solutions at the origin as limits of $k T$-periodic solutions (subharmonic solutions) as $k \rightarrow \infty$, or alternatively, as critical points of the functional $I(u)$ over a suitable space of functions on the whole space $\mathbb{R}$ (typically, $H^{1}\left(\mathbb{R}, \mathbb{R}^{N}\right)$ ).

For singular systems, one assumes that $V \in C^{1}\left(\mathbb{R} \times \mathbb{R}^{N} \backslash S\right)$ and $\lim _{u \rightarrow S}|V(t, u)|=\infty$ for some $S \subset \mathbb{R}^{N}$. Although the study of singular systems is perhaps as old as Kepler's classical problem in mechanics,

$$
\ddot{u}+\frac{u}{|u|^{3}}=0
$$


(and, also, the $\mathrm{N}$-body problem), the interest in such problems was renewed by the pioneering papers [2] of Gordon in 1975 and [3] of Rabinowitz in 1978. In [2], the notion of strong force is introduced to deal with singular problems, while in [3], the use of variational methods is brought into the study of periodic solutions of Hamiltonian systems.

The present paper is concerned with the existence of homoclinic solutions for the following second order nonautonomous singular Hamiltonian systems:

$$
\ddot{u}+a(t) W_{u}(u)=0,
$$

where $-\infty<t<+\infty, u=\left(u_{1}, u_{2}, \ldots, u_{N}\right) \in \mathbb{R}^{N}(N \geq 3), a$ : $\mathbb{R} \rightarrow \mathbb{R}$ is a continuous bounded function, and the potential $W: \mathbb{R}^{N} \backslash\{\xi\} \rightarrow \mathbb{R}$ has a singularity at $0 \neq \xi \in \mathbb{R}^{N} ; W_{u}(u)$ is the gradient of $W$ at $u$. We recall that a homoclinic solution of (HS) is a solution such that $u(t) \in \mathbb{R}^{N} \backslash\{\xi\}$ for all $t \in \mathbb{R}$ and

$$
u(t), \quad \dot{u}(t) \longrightarrow 0 \quad \text { as } t \longrightarrow \pm \infty .
$$

Throughout this paper, we assume that the following hypotheses are supposed:

(A) $a: \mathbb{R} \rightarrow \mathbb{R}$ is a continuous function such that

$$
0<a_{0} \leq a(t) \leq a_{\infty} \quad \forall t \in \mathbb{R},
$$

where $a_{0}$ and $a_{\infty}$ are two positive constants and

$$
a^{\prime}(t)<0 \quad \forall t \in \mathbb{R}
$$

$\left(\mathrm{W}_{1}\right) W \in C^{2}\left(\mathbb{R}^{N} \backslash\{\xi\}, \mathbb{R}\right)$ for some $\xi \in \mathbb{R}^{N} \backslash\{0\}$;

$\left(\mathrm{W}_{2}\right) W(u) \leq 0$ for all $u \in \mathbb{R}^{N} \backslash\{\xi\}$ and $W(u)=0$ if and only if $u=0$ and $\lim _{|u|} \rightarrow \infty \sup W(u) \equiv \bar{W}<0$;

$\left(\mathrm{W}_{3}\right)$ there is a constant $\delta \in(0,(1 / 2)|\xi|)$ such that

$$
W(u)+\frac{1}{2}\left(W_{u}(u), u\right) \leq 0
$$

for all $u \in B_{\delta}(0)$, where $B_{\delta}(0)=\left\{u \in \mathbb{R}^{N}:|u|<\delta\right\} ;$

$\left(\mathrm{W}_{4}\right) \lim _{u \rightarrow \xi} W(u)=-\infty$;

$\left(\mathrm{W}_{5}\right)$ there is a neighbourhood $\Omega$ of $\xi$ in $\mathbb{R}^{N}$ and a function $U \in C^{1}(\Omega \backslash\{\xi\}, \mathbb{R})$ such that $U(u) \rightarrow \infty$ as $u \rightarrow \xi$ and

$$
-W(u) \geq|\nabla U(u)|^{2} \quad \forall u \in \Omega \backslash\{\xi\} .
$$

Now we are in the position to state our main result.

Theorem 1. Under the conditions of $(A)$ and $\left(W_{1}\right)-\left(W_{5}\right),(H S)$ has at least one nontrivial homoclinic solution.

Remark 2. The assumption $\left(\mathrm{W}_{5}\right)$ is the so-called strong force condition (see Gordon [2]), which is used to verify the PalaisSmale condition for the functional corresponding to the approximating problem (HS : T) defined below. For example, $\left(\mathrm{W}_{5}\right)$ is satisfied when $W(u)=-|u-\xi|^{-\alpha}(\alpha \geq 2)$ in a neighbourhood of $\xi$. The assumption $\left(\mathrm{W}_{3}\right)$ is a kind of concavity condition for $W(u)$ near 0 . In particular, $\left(\mathrm{W}_{3}\right)$ holds for small $\delta>0$ when $W^{\prime \prime}(0)$ is negative definite.
In the case of autonomous singular Hamiltonian systems, the first result on existence of a homoclinic orbit using variational methods was obtained by Tanaka [4] under essentially the same assumptions as above. In [4], Tanaka used a minimax argument from Bahri and Rabinowitz [5] in order to get approximating solutions of the following boundary value problem:

$$
\begin{gathered}
\ddot{u}+\nabla V(u)=0, \quad t \in(0, m), \\
u(0)=u(m)
\end{gathered}
$$

as critical points of the corresponding functional and obtained uniform estimates to show that those solutions converge weakly to a nontrivial homoclinic solution of (HS). Regarding multiplicity of homoclinics, still in the autonomous singular case, early results were obtained by Caldiroli [6], who showed existence of two homoclinic orbits, and by Bessi [7], who used Lyusternick-Schnirelman category to prove the existence of $N-1$ distinct homoclinics for potentials satisfying a pinching condition. For $N=3$, Janczewska and Maksymiuk in [8], via use of variational methods and geometrical arguments, investigated the existence of homoclinic orbits. In addition, different kinds of multiplicity results were obtained in $[9,10]$ (still for conservative systems) by exploiting the topology of $\mathbb{R}^{N} \backslash S$, the domain of the potential, when the set $S$ is such that the fundamental group of $\mathbb{R}^{N} \backslash S$ is nontrivial.

In the case of planar autonomous systems, more extensive existence and multiplicity results were obtained. Indeed, under essentially the same conditions as above with $N=2$, Rabinowitz showed in [11] that (HS) has at least a pair of homoclinic solutions by exploiting the topology of the plane and minimizing the energy functional on classes of sets with a fixed winding number around the singularity $\xi$. The result in [11] was substantially improved in [12] where, using the same idea, the authors showed that a nondegeneracy variational condition introduced in [12] is in fact necessary and sufficient for the minimum problem to have a solution in the class of sets with winding number greater than 1 and, therefore, proved a result on existence of infinitely many homoclinic solutions. For the recent results, we refer the reader to $[13,14]$.

On the other hand, in the case of $T$-periodic time dependent Hamiltonians in $\mathbb{R}^{N}$, existence of infinitely many homoclinic orbits was obtained for smooth Hamiltonians by using a variational procedure due to Séré in $[15,16]$ for the first systems and in $[17,18]$ for second order systems. In case $N=2$, using theses ideas, Rabinowitz [19] constructed infinitely many multibump homoclinic solutions for $V(t, u)$ of the form $a(t) W(u)$, with $a(t)$ being almost periodic and $W$ satisfying $(\mathrm{A})$ and $\left(\mathrm{W}_{1}\right)-\left(\mathrm{W}_{5}\right)$. Recently, Izydorek and Janczewska in [20] investigated the existence of at least two connecting orbits in some more general sense. For the case that $N \geq 3$, recently, the authors in [21], using the category theory, for the first time considered the existence of infinitely many geometrically distinct homoclinic solutions, under the assumptions that $a(t)$ is periodic and $W$ satisfies $\left(\mathrm{W}_{1}\right)-\left(\mathrm{W}_{5}\right)$ and the following condition on $W$ at infinity: 
$\left(\mathrm{W}_{6}\right)$ there exists $U_{\infty} \in C\left(\mathbb{R}^{N} \backslash B_{R_{0}}, \mathbb{R}\right)$ such that $\lim _{|u| \rightarrow \infty}\left|U_{\infty}(u)\right|=\infty$ and $W(u) \leq-\left|\nabla U_{\infty}(u)\right|^{2}$ for $u$ large.

Here we must point out that all the results mentioned above are obtained for the case that (HS) is autonomous or periodic or almost periodic. Motivated mainly by the works of $[4,21]$, in present paper, we focus our attention on the case that $N \geq 3$ and (HS) is non-autonomous (neither periodic nor almost periodic). To the best of our knowledge, this is the first result on the existence of homoclinic solutions for second order nonautonomous singular Hamiltonian systems in $\mathbb{R}^{N}$ $(N \geq 3)$. The proof of Theorem 1 will be demonstrated in the following sections. To this end, we employ the technique used in [4]. Explicitly, considering the approximating problem:

$$
\begin{gathered}
\ddot{u}+a(t) W_{u}(u)=0, \quad t \in(0, T) \\
u(0)=u(T)=0,
\end{gathered}
$$

solutions of (HS : T) are obtained as critical points of the functional $I_{T}(u)$ defined in Section 2 . We show the existence of critical points of $I_{T}(u)$ via a minimax argument, which is essentially due to Bahri and Rabinowitz [5]. Furthermore, we also get some estimates, which are uniform with respect to $T \geq 1$, for minimax values and corresponding critical points $u(t ; T)$. These uniform estimates permit us to let $T \rightarrow \infty$; for a suitable sequence $\left\{\tau_{k}\right\}_{k=1}^{\infty}$ and a subsequence $T_{k} \rightarrow \infty$, we see that $u\left(t+\tau_{k} ; T_{k}\right)$ converges weakly to a homoclinic solution of (HS) as $k \rightarrow \infty$.

The remaining part of this paper is organized as follows. In Section 2, via a minimax argument, for any $T \geq 1$, we show that (HS: T) has at least one nontrivial solution $u(t ; T)$. In Section 3, some uniform estimates for solutions $u(t ; T)$ are obtained to investigate homoclinic solution of (HS). In Section 4, we are devoted to accomplishing the proof of Theorem 1 .

\section{Approximating Problem (HS : T)}

In this section, we investigate the approximating problem (HS : T) via a minimax argument. Denote by $H_{0}^{1}\left(0, T ; \mathbb{R}^{N}\right)$ the usual Sobolev space on $(0, T)$ with values in $\mathbb{R}^{N}$ under the norm

$$
\|u\|=\left(\int_{0}^{T}|\dot{u}|^{2} d t\right)^{1 / 2} .
$$

Let

$$
\Lambda_{T}=\left\{u \in H_{0}^{1}\left(0, T ; \mathbb{R}^{N}\right): u(t) \neq \xi \forall t \in[0, T]\right\} .
$$

Clearly, $\Lambda_{T}$ is an open subset of $H_{0}^{1}\left(0, T ; \mathbb{R}^{N}\right)$. Define the functional $I_{T}: \Lambda_{T} \rightarrow \mathbb{R}$ as follows:

$$
I_{T}(u)=\frac{1}{2} \int_{0}^{T}|\dot{u}(t)|^{2} d t-\int_{0}^{T} a(t) W(u(t)) d t .
$$

Under the assumptions of Theorem 1, as usual, one can show that $I_{T} \in C^{1}\left(\Lambda_{T}, \mathbb{R}\right)$ and

$$
\begin{aligned}
I_{T}(u) v= & \int_{0}^{T}(\dot{u}(t), \dot{v}(t)) d t \\
& -\int_{0}^{T} a(t)\left(W_{u}(u(t)), v(t)\right) d t, \quad \forall u, v \in \Lambda_{T} .
\end{aligned}
$$

Then there is an one-to-one correspondence between critical points of $I_{T}$ and classical solutions of (HS : T).

In order to obtain a critical point of $I_{T}$, we use a minimax argument. To do so, $I_{T}$ must satisfy the Palais-Smale condition ((PS) condition) on $\Lambda_{T}$; that is, for any sequence $\left\{u_{j}\right\}_{j=1}^{\infty} \subset \Lambda_{T}$ such that $I_{T}\left(u_{j}\right)$ is bounded and $I_{T}^{\prime}\left(u_{j}\right) \rightarrow 0$ as $j \rightarrow \infty,\left\{u_{j}\right\}_{j=1}^{\infty}$ possesses a subsequence converging to some $u \in \Lambda_{T}$.

Proposition 3. If a and $W$ satisfy $(A),\left(W_{1}\right),\left(W_{2}\right),\left(W_{4}\right)$, and $\left(W_{5}\right)$, then $I_{T}$ satisfies the (PS) condition.

Proof. Let $\left\{u_{j}\right\}_{j=1}^{\infty} \subset \Lambda_{T}$ be a sequence such that $I_{T}\left(u_{j}\right)$ is bounded and $I^{\prime}\left(u_{j}\right) \rightarrow 0$ as $j \rightarrow \infty$. Then, by $\left(\mathrm{W}_{2}\right)$ and the definition of $I_{T},\left\{u_{j}\right\}_{j=1}^{\infty}$ is bounded in $H_{0}^{1}(0, T ; \mathbb{R})$. Hence, we can extract a subsequence of $\left\{u_{j}\right\}_{j=1}^{\infty}$, still denoted by $\left\{u_{j}\right\}_{j=1}^{\infty}$, such that $u_{j}$ converges to $u \in \overline{\Lambda_{T}}$ (the closure of $\Lambda_{T}$ in $\left.H_{0}^{1}\left(0, T ; \mathbb{R}^{N}\right)\right)$ weakly in $H_{0}^{1}\left(0, T ; \mathbb{R}^{N}\right)$. On the other hand, by Lemma 2.1 in [22], if $u \in \partial \Lambda_{T}$, then

$$
-\int_{0}^{T} a(t) W\left(u_{j}(t)\right) d t \longrightarrow \infty \quad \text { as } j \longrightarrow \infty .
$$

That is, $I_{T}\left(u_{j}\right) \rightarrow \infty$. Hence, $u \in \Lambda_{T}$. Subsequently, in view of $\left(\mathrm{W}_{1}\right)$ and the Sobolev compact embedding theorem, it follows that $u_{j} \rightarrow u$ strongly in $H_{0}^{1}\left(0, T ; \mathbb{R}^{N}\right)$.

Since $I_{T}$ satisfies the (PS) condition, we have the following deformation theorem (see [23]).

Lemma 4. Suppose that $c$ is not a critical value of $I_{T}$. Then, for all $\bar{\varepsilon}$, there are an $\varepsilon \in(0, \bar{\varepsilon})$ and $\eta \in C\left([0, T] \times \Lambda_{T}, \Lambda_{T}\right)$ such that

(1) $\eta(1, u)=u$ if $I_{T}(u) \notin(c-\bar{\varepsilon}, c+\bar{\varepsilon})$;

(2) $I_{T}(\eta(\tau, u)) \leq I_{T}(u)$ for all $(\tau, u) \in[0,1] \times \Lambda_{T}$;

(3) $\eta\left(1, I_{T}^{c+\varepsilon}\right) \subset I_{T}^{c-\epsilon}$,

where $I_{T}^{b}$ denotes the level set defined by

$$
I_{T}^{b}=\left\{u \in \Lambda_{T}: I_{T}(u) \leq b\right\} .
$$

Now, one introduces a minimax procedure for $I_{T}$. Let

$$
\begin{gathered}
D^{N-2}=\left\{x \in \mathbb{R}^{N}:|x| \leq 1\right\}, \\
\Gamma_{T}=\left\{\gamma \in C\left(D^{N-2}, \Lambda_{T}\right):\right. \\
\left.\gamma(x)(t)=0 \forall x \in \partial D^{N-2}, t \in[0, T]\right\} .
\end{gathered}
$$


For $\gamma \in \Gamma_{T}$, one observes that $\gamma(x)(t)=0$ for all

$$
\begin{aligned}
(x, t) & \in\left(\partial D^{N-2} \times[0, T]\right) \cup\left(D^{N-2} \times\{0, T\}\right) \\
& =\partial\left(D^{N-2} \times[0, T]\right) .
\end{aligned}
$$

Since $D^{N-2} \times[0, T] / \partial\left(D^{N-2} \times[0, T]\right) \simeq S^{N-1}$, one can consider for each $\gamma \in \Lambda_{T}$ a map $\tilde{\gamma}: S^{N-1} \rightarrow S^{N-1}$ defined by

$$
\tilde{\gamma}(x, t)=\frac{\gamma(x)(t)-\xi}{|\gamma(x)(t)-\xi|} .
$$

One denotes by $\operatorname{deg} \tilde{\gamma}$ the Brouwer degree of a map $\tilde{\gamma}$ : $S^{N-1} \rightarrow S^{N-1}$. Let

$$
\Gamma_{T}^{*}=\left\{\gamma \in \Lambda_{T}: \operatorname{deg} \widetilde{\gamma} \neq 0\right\} .
$$

It is obvious that $\Gamma_{T}^{*} \neq \emptyset$. Define a minimax value of $I_{T}$ by

$$
c(T)=\inf _{\gamma \in \Gamma_{T}^{*}} \sup _{x \in D^{N-2}} I_{T}(\gamma(x)) .
$$

Then one has the following.

Proposition 5. $c(T)>0$ is a critical value of $I_{T}$.

Proof. $c(T)>0$ will be seen later in Proposition 6. Here we assume it and prove that $c(T)$ is a critical value of $I_{T}$. On the contrary, we suppose that $c(T)>0$ is not a critical value. Taking $c=c(T)>0$ and $\bar{\varepsilon}=c / 2$ in Lemma 4, we have a deformation flow $\eta(\tau, u)$ with the properties (1)-(3). Moreover, we can verify

$$
\eta\left(1, \Gamma_{T}^{*}\right) \subset \Gamma_{T}^{*}
$$

In fact, since $\eta(1,0)=0$ (see (1)), we have $\eta(1, \gamma(x))=0$ for $x \in \partial D^{N-2}$. On the other hand, due to (2), we have

$$
I_{T}(\eta(\tau, \gamma(x))) \leq I_{T}(\gamma(x))<\infty \quad \forall \gamma \in \Gamma_{T}^{*}, \tau \in[0,1] .
$$

Hence, $\eta(\tau, \gamma(x))(t) \neq \xi$ for all $(x, t) \in D^{N-2} \times[0, T]$ and $\tau \in$ $[0,1]$. Thus we have

$$
\operatorname{deg} \eta(1, \gamma)=\operatorname{deg} \eta(0, \gamma)=\operatorname{deg} \tilde{\gamma} \neq 0 .
$$

Therefore, $\eta(1, \gamma) \in \Gamma_{T}^{*}$ for $\gamma \in \Gamma_{T}^{*}$; that is, (22) holds.

Choose $\gamma \in \Gamma_{T}^{*}$ such that $\max _{x \in D^{N-2}} I_{T}(\gamma(x)) \leq c+\varepsilon$ and consider $\eta(1, \gamma(x)) \in \Gamma_{T}^{*}$. Then, in virtue of (3), we obtain

$$
\max _{x \in D^{N-2}} I_{T}(\eta(1, \gamma(x))) \leq c-\varepsilon
$$

which contradicts the definition of $c=c(T)$. Therefore, $c(T)>0$ is a critical value of $I_{T}$.

Proposition 6. There is a constant $c_{0}>0$ independent of $T \geq$ 1 such that

$$
0<c_{0} \leq c(T) \leq c(1) \quad \forall T \geq 1 .
$$

Proof. For any given $\gamma \in \Gamma_{1}^{*}$, we define $\gamma^{T} \in \Gamma_{T}(T \geq 1)$ by

$$
\gamma^{T}(x)(t)= \begin{cases}\gamma(x)(t), & (x, t) \in D^{N-2} \times[0,1], \\ 0, & (x, t) \in D^{N-2} \times(1, T] .\end{cases}
$$

Then, we can easily see the following:

(1) $\operatorname{deg} \widetilde{\gamma}^{T}=\operatorname{deg} \widetilde{\gamma} \neq 0$, that is, $\gamma^{T} \in \Gamma_{T}^{*}$ for all $\gamma \in \Gamma_{1}^{*}$;

(2) $I_{T}\left(\gamma^{T}(x)\right)=I_{1}(\gamma(x))$ for all $x \in D^{N-2}$ and $\gamma \in \Gamma_{1}^{*}$.

Therefore, we get

$$
\begin{aligned}
c(T) & =\inf _{\gamma \in \Gamma_{T}^{*}} \max _{x \in D^{N-2}} T_{T}(\gamma(x)) \\
& \leq \inf _{\gamma \in \Gamma_{1}^{*}} \max _{x \in D^{N-2}} T_{T}\left(\gamma^{T}(x)\right) \\
& =\inf _{\gamma \in \Gamma_{1}^{*}} \max _{x \in D^{N-2}} T_{1}(\gamma(x)) \\
& =c(1) .
\end{aligned}
$$

In what follows, we prove the existence of a constant $c_{0}>0$ such that $c(T) \geq c_{0}>0$ for all $T \geq 1$. For any given $\gamma \in \Gamma_{T}^{*}$, we have

$$
\left\{\gamma(x)(t):(x, t) \in D^{N-2} \times[0, T]\right\} \cap\left(\mathbb{R}^{N} \backslash B_{2 \delta}(0)\right) \neq \emptyset,
$$

where $\delta>0$ is defined in $\left(\mathrm{W}_{3}\right)$. Otherwise, we can easily observe that $\operatorname{deg} \tilde{\gamma}=0$. Hence, there is $\left(x_{0}, t_{0}\right) \in D^{N-2} \times[0, T]$ such that

$$
\gamma\left(x_{0}\right)\left(t_{0}\right) \notin B_{2 \delta}(0) .
$$

Since $\gamma\left(x_{0}\right)\left(t_{0}\right)=0$, there is an $s_{0} \in\left(0, t_{0}\right)$ such that

$$
\gamma\left(x_{0}\right)\left(s_{0}\right) \in \partial B_{\delta}(0) \quad \gamma\left(x_{0}\right)(t) \notin B_{\delta}(0) \quad \forall t \in\left(s_{0}, t_{0}\right) .
$$

By the Schwarz inequality, we have for $u(t)=\gamma\left(x_{0}\right)(t)$

$$
\begin{aligned}
I_{T}\left(\gamma\left(x_{0}\right)\right) & \geq \int_{s_{0}}^{t_{0}} \frac{1}{2}|\dot{u}(t)|^{2} d+\int_{s_{0}}^{t_{0}}-a(t) W(u(t)) d t \\
& \geq \frac{1}{2\left(t_{0}-s_{0}\right)}\left(\int_{s_{0}}^{t_{0}}|\dot{u}(t)| d t\right)^{2}+a_{0} m_{\delta}\left(t_{0}-s_{0}\right) \\
& \geq \frac{1}{2\left(t_{0}-s_{0}\right)}\left|u\left(t_{0}\right)-u\left(s_{0}\right)\right|^{2}+a_{0} m_{\delta}\left(t_{0}-s_{0}\right) \\
& \geq\left(2 a_{0} m_{\delta}\right)^{1 / 2}\left|u\left(t_{0}\right)-u\left(s_{0}\right)\right| \\
& \geq\left(2 a_{0} m_{\delta}\right)^{1 / 2} \delta \equiv c_{0}
\end{aligned}
$$

where

$$
m_{\delta}=\min _{x \in \mathbb{R}^{N} \backslash B_{\delta}(0)}-W(x)>0 .
$$


Thus, we have

$$
\max _{x \in D^{N-2}} I_{T}(\gamma(x)) \geq c_{0} \quad \forall \gamma \in \Gamma_{T}^{*},
$$

which yields that

$$
c(T) \geq c_{0} .
$$

Combining with (28) and (35), we obtain the desired conclusion.

In view of Propositions 5 and 6, we deduce the following proposition concerned with the uniform boundness of critical value of (HS : T).

Proposition 7. For $T \geq 1$, (HS: T) possesses a solution $u(t ; T)$ such that

$$
0<c_{0} \leq I_{T}(u(t ; T)) \leq c_{1}<\infty \quad \forall T \geq 1,
$$

where $c_{0}$ and $c_{1}>0$ are independent of $T \geq 1$.

\section{Estimates for Solutions of (HS : T)}

In this section, we give some estimates on the solutions $u(\cdot ; T)$ of (HS : T) to allow $T$ go to $\infty$ in Section 4. Firstly, from the definition of $I_{T}$ and Proposition 7, we have the following.

Lemma 8. There is a constant $C>0$ which is independent of $T \geq 1$ such that

$$
\|\dot{u}(\cdot ; T)\|_{L^{2}(0, T)}, \quad \int_{0}^{T}-a(t) W(u(t ; T)) d t \leq C \quad \forall T \geq 1 .
$$

In what follows, we denote by $C, C^{\prime}, \ldots$, various constants which are independent of $T \geq 1$.

Proposition 9. Consider $\|u(\cdot ; T)\|_{L^{\infty}(0, T)} \leq C$ for all $T \geq 1$.

Proof. Suppose that $u(t ; T) \notin \bar{B}_{\delta}(0)$ for some $t \in(0, T)$. On account of $u(0 ; T)=0$, we can find an interval $(s, t) \subset(0, t)$ such that $u(s ; T) \in \partial B_{\delta}(0)$ and $u(\tau ; T) \notin B_{\delta}(0)$ for all $\tau \in(s, t)$, which, combining with Lemma 8 , implies that

$$
\begin{aligned}
\int_{s}^{t}|\dot{u}(\tau ; T)| d \tau & \leq(t-s)^{1 / 2}\left(\int_{s}^{t}|\dot{u}(\tau ; T)|^{2} d \tau\right)^{1 / 2} \\
& \leq(t-s)^{1 / 2}\|\dot{u}(\cdot ; T)\|_{L^{2}(0, T)} \\
& \leq C(t-s)^{1 / 2} .
\end{aligned}
$$

On the other hand, we have

$$
\begin{aligned}
t-s & \leq \frac{1}{a_{0} m_{\delta}} \int_{s}^{t}-a(\tau) W(u(\tau ; T)) d \tau \\
& \leq \frac{1}{a_{0} m_{\delta}} \int_{0}^{T}-a(\tau) W(u(\tau ; T)) d \tau \leq C^{\prime},
\end{aligned}
$$

where $m_{\delta}$ is defined in (33). Combining with (38) and (39), we deduce that

$$
\int_{s}^{t}|\dot{u}(\tau ; T)| d \tau \leq C^{\prime \prime}
$$

which yields that

$$
|u(t ; T)| \leq|u(s ; T)|+\int_{s}^{t}|\dot{u}(\tau ; T)| d \tau \leq \delta+C^{\prime \prime} .
$$

As a result, we conclude that

$$
\|u(\cdot ; T)\|_{L^{\infty}(0, T)} \leq \delta+C^{\prime \prime}
$$

Lemma 10. Define the function $E_{T}(t)$ as follows:

$$
E_{T}(t)=\frac{1}{2}|\dot{u}(t ; T)|^{2}+a(t) W(u(t ; T)) .
$$

Then, $E_{T}(t)$ is nondecreasing on $[0, T]$. Furthermore, one has

$$
|\dot{u}(0 ; T)| \longrightarrow 0, \quad|\dot{u}(T ; T)| \longrightarrow 0 \quad \text { as } T \longrightarrow \infty .
$$

Proof. In view of $(\mathrm{A})$ and $\left(\mathrm{W}_{2}\right)$, it deduces that

$$
\begin{aligned}
\frac{d E_{T}(t)}{d t}= & (\ddot{u}(t ; T), \dot{u}(t ; T))+\left(a(t) W_{u}(u(t ; T)), \dot{u}(t ; T)\right) \\
& +a^{\prime}(t) W(t(t ; T)) \\
= & a^{\prime}(t) W(u(t ; T)) \geq 0,
\end{aligned}
$$

which implies that $E_{T}(t)$ is nondecreasing on $[0, T]$. Thus, we have $E_{T}(t) \geq|\dot{u}(0 ; T)|^{2} / 2$ for all $t \in[0, T]$. Integrating (43) over $(0, T)$ and by Lemma 8 , we have

$$
\begin{aligned}
\frac{T}{2}|\dot{u}(0 ; T)|^{2} \leq & \int_{0}^{T} E_{T}(t) d t \\
= & \frac{1}{2}\|\dot{u}(\cdot ; T)\|_{L^{2}(0, T)} \\
& -\int_{0}^{T}-a(t) W(u(t ; T)) d t \leq C
\end{aligned}
$$

for all $T \geq 1$. We observe the fact that $|\dot{u}(0 ; T)|>0$. Otherwise, $\dot{u}(0 ; T)=0$ and then we have $u(t ; T) \equiv 0$ by the uniquemess of the solution of the initial value problem:

$$
\begin{gathered}
\ddot{u}+a(t) W(u)=0, \\
u(0)=\dot{u}(0)=0,
\end{gathered}
$$

which contradicts the fact that $I_{T}(u(t ; T))=c(T)>0$. Consequently, combining with $u(0 ; T)=0$ and in view of (46), we have

$$
|\dot{u}(0 ; T)| \longrightarrow 0 \quad \text { as } T \longrightarrow \infty \text {. }
$$


It only remains to show that $|\dot{u}(T ; T)| \rightarrow 0$ as $T \rightarrow \infty$. On the contrary, due to the facts that $E_{T}(t)$ is increasing on $[0, T], E_{T}(T)=(1 / 2)|\dot{u}(T ; T)|^{2}$, and

$$
\begin{aligned}
& \dot{u}(T ; T)-\dot{u}(0 ; T) \\
& \quad=\int_{0}^{T} \ddot{u}(t ; T) d t=-\int_{0}^{T} a(t) W(u(t ; T)) d t \leq C,
\end{aligned}
$$

it occurs that

$$
\begin{aligned}
0<\lim _{T \rightarrow \infty} E_{T}(T) & =\lim _{T \rightarrow \infty} \frac{1}{2}|\dot{u}(T ; T)|^{2}+a(T) W(u(T ; T)) \\
& =\lim _{T \rightarrow \infty} \frac{1}{2}|\dot{u}(T ; T)|^{2}=E_{\infty}<\infty .
\end{aligned}
$$

However, this contradicts the fact that $\|\dot{u}(\cdot ; T)\|_{L^{2}(0, T)} \leq C$ for all $T \geq 1$.

The following proposition gives us an $L^{\infty}$-bound from below on $u(t ; T)$ for all $T \geq 1$. Here, it must be pointed that the condition $\left(\mathrm{W}_{3}\right)$ is used only in this proposition.

Proposition 11. Consider $\|u(\cdot ; T)\|_{L^{\infty}(0, T)} \geq \delta$ for all $T \geq 1$.

Proof. Using (43), we get

$$
\begin{aligned}
\frac{1}{2} \frac{d^{2}}{d t^{2}}|u(t ; T)|^{2}= & \frac{d}{d t}(\dot{u}(t ; T), u(t ; T)) \\
= & |\dot{u}(t ; T)|^{2}-\left(a(t) W_{u}(u(t ; T)), u(t ; T)\right) \\
= & -2 a(t) W(u(t ; T)) \\
& -a(t)\left(W_{u}(u(t ; T)), u(t ; T)\right)+2 E_{T} .
\end{aligned}
$$

Due to the fact that $(1 / 2)|\dot{u}(0 ; T)|^{2}>0$ (the reason has been explained in Lemma 10) and the condition $\left(\mathrm{W}_{3}\right)$, we obtain that

$$
\frac{d^{2}}{d t^{2}}|u(t ; T)|^{2}>0 \quad \text { whenever } u(t ; T) \in B_{\delta}(0) .
$$

Suppose that $|u(t ; T)|^{2}$ takes its maximum at $t_{0} \in(0, T)$. From the above inequality, we deduce that $u\left(t_{0} ; T\right) \notin B_{\delta}(0)$. Thus, we have

$$
\|u(\cdot ; T)\|_{L^{\infty}(0, T)} \geq \delta \quad \forall T \geq 1 .
$$

By Proposition 11, we can find two numbers $0<\tau_{T}^{1} \leq \tau_{T}^{2}<$ $T$ such that $u\left(\tau_{1} ; T\right), u\left(\tau_{2} ; T\right) \in \partial B_{\delta}(0)$, and $u(t ; T) \in B_{\delta}(0)$ for all $t \in\left[0, \tau_{T}^{1}\right] \cup\left[\tau_{T}^{2}, T\right]$. For $\tau_{T}^{1}$ and $\tau_{T}^{2}$, we need the following property.

Lemma 12. Consider $\tau_{T}^{1}, T-\tau_{T}^{2} \rightarrow \infty$ as $T \rightarrow \infty$.
Proof. Let $u_{\sigma}(t)$ be a solution of the following initial value problem:

$$
\begin{gathered}
\ddot{u}+a(t) W_{u}(u)=0, \\
u(0)=0, \quad \dot{u}(0)=\sigma .
\end{gathered}
$$

By the continuous dependence of $u_{\sigma}(t)$ on the initial data, for any $l>0$, there is an $\epsilon>0$ such that

$$
u_{\sigma}(t) \in B_{\delta}(0) \quad \text { for } t \in[0, l],|\sigma| \leq \epsilon .
$$

By (44), for any $l>0$, we can find $T_{l} \geq l$ such that

$$
u(t ; T) \in B_{\delta}(0) \quad \text { for } t \in[0, l], T \geq T_{l} .
$$

That is, $\tau_{T}^{1} \geq l$ for $T \geq T_{l}$. Therefore, we have $\tau_{T}^{1} \rightarrow \infty$ as $T \rightarrow \infty$. Similarly, we can obtain $T-\tau_{T}^{2} \rightarrow \infty$ as $T \rightarrow$ $\infty$.

\section{Proof of Theorem 1}

In this section, we construct a homoclinic solution of (HS) as a limit of the solutions $u(t ; T)$ of (HS : T) when $T$ goes to $\infty$ and complete the proof of Theorem 1 . In order to accomplish such a process, for each $T \geq 1$, we define $\widetilde{u}(t ; T) \in H^{1}\left(\mathbb{R}, \mathbb{R}^{N}\right)$ by

$$
\widetilde{u}(t ; T)= \begin{cases}u\left(t+\tau_{T}^{1}\right), & t \in\left[-\tau_{T}^{1}, T-\tau_{T}^{1}\right] \\ 0, & \text { otherwise. }\end{cases}
$$

Then it directly follows from Lemma 8 and Proposition 9 that

(i) $\widetilde{u}(t ; T)$ is a solution of $(\mathrm{HS})$ in $\left(-\tau_{T}^{1}, T-\tau_{T}^{1}\right)$;

(ii) $\widetilde{u}(0 ; T) \in \partial B_{\delta}(0)$ for all $T \geq 1$;

(iii) $\|\widetilde{u}(\cdot ; T)\|_{L^{2}\left(\mathbb{R}, \mathbb{R}^{N}\right)},\|\widetilde{u}(\cdot ; T)\|_{L^{\infty}\left(\mathbb{R}, \mathbb{R}^{N}\right)}$, and $\int_{\mathbb{R}}-a(t) W(\widetilde{u}$ $(t ; T)) d t$ are uniformly bounded for $T \geq 1$.

By (iii), we can extract a subsequence $T_{k} \rightarrow \infty$ such that $\widetilde{u}\left(t ; T_{k}\right)$ converges to some $y(t) \in C\left(\mathbb{R}, \mathbb{R}^{N}\right) \cap L^{\infty}\left(\mathbb{R}, \mathbb{R}^{N}\right)$ with $y(t) \in L^{2}\left(\mathbb{R}, \mathbb{R}^{N}\right)$ in the following sense:

$$
\begin{gathered}
\tilde{u}\left(t ; T_{k}\right) \longrightarrow y(t) \quad \text { in } L_{\text {loc }}^{\infty}\left(\mathbb{R}, \mathbb{R}^{N}\right), \\
\dot{\tilde{u}}\left(t ; T_{k}\right) \rightarrow \dot{y}(t) \quad \text { weakly in } L^{2}\left(\mathbb{R}, \mathbb{R}^{N}\right) .
\end{gathered}
$$

Moreover, we have

$$
\begin{aligned}
& \int_{\mathbb{R}}-a(t) W(y(t)) d t \\
& \quad \leq \lim _{T_{k} \rightarrow \infty} \sup \int_{\mathbb{R}}-a(t) W\left(\widetilde{u}\left(t ; T_{k}\right)\right) d t \leq C<\infty .
\end{aligned}
$$


Due to the strong force condition $\left(\mathrm{W}_{5}\right)$, similar to Lemma 2.1 in [22], we can also obtain that

$$
y(t) \neq \xi \quad \forall t \in \mathbb{R} .
$$

In what follows, we focus our attentions to show that $y(t)$ is exactly right the homiclinic solution of (HS) that we need.

Proposition 13. $y(t)$ is a nontrivial solution of $(H S)$ on $\mathbb{R}$.

Proof. In view of (61), it is sufficient to verify that for any $\psi \in$ $C_{0}^{\infty}\left(\mathbb{R}, \mathbb{R}^{N}\right)$

$$
\int_{\mathbb{R}}\left[(\dot{y}(t), \dot{\psi}(t))-a(t)\left(W_{u}(y(t)), \psi(t)\right)\right] d t=0 .
$$

By Lemma 12, we can choose $k_{0} \in \mathbb{N}$ such that supp $\psi \subset$ $\left(-\tau_{T_{k}}^{1}, T-\tau_{T_{k}}^{1}\right)$ for all $k \geq k_{0}$. By property (i) of $\tilde{u}(t ; T)$, we have for all $k \geq k_{0}$

$$
\int_{\mathbb{R}}\left[\left(\dot{\tilde{u}}\left(t ; T_{k}\right), \dot{\psi}(t)\right)-a(t)\left(W_{u}\left(\widetilde{u}\left(t ; T_{k}\right)\right), \psi(t)\right)\right] d t=0 .
$$

On account of (58) and (59), we get (62) as $k \rightarrow \infty$. On the other hand, as a direct consequence of (58) and the property (ii) of $\widetilde{u}(t ; T)$, we have $y(0) \in \partial B_{\delta}(0)$; that is, $y(t)$ is nontrivial.

As the last step of the proof of Theorem 1, we show that $y(t)$ and $\dot{y}(t)$ satisfy the following property.

Proposition 14. Consider $y(t), \dot{y}(t) \rightarrow 0$ as $t \rightarrow \pm \infty$.

Proof. Here, we just check the case that $t \rightarrow \infty$, since it is the same as $t \rightarrow-\infty$. First, we prove $y(t) \rightarrow 0$ as $t \rightarrow \infty$. On the contrary, we assume that $y(t) \nrightarrow 0$ as $t \rightarrow \infty$. Then, for some sequence $t_{k} \rightarrow \infty$ and for some $\epsilon>0$, we have

$$
y\left(t_{k}\right) \notin B_{\epsilon}(0) \quad \forall k \in \mathbb{N} \text {. }
$$

On the other hand, in view of $\left(\mathrm{W}_{2}\right)$ and $(60)$, it follows that

$$
\text { meas }\left\{t \in \mathbb{R}: y(t) \notin B_{\epsilon / 2}(0)\right\}<\infty \text {. }
$$

Hence, there is a sequence $\bar{t}_{k} \rightarrow \infty$ such that $y\left(\bar{t}_{k}\right) \in B_{\epsilon / 2}(0)$. Thus, $y(t)$ must intersect $\partial B_{\epsilon}(0)$ and $\partial B_{\epsilon / 2}(0)$ infinitely as $t \rightarrow$ $\infty$. However, this contradicts $\dot{y}(t) \in L^{2}\left(\mathbb{R}, \mathbb{R}^{N}\right)$ and $(60)$. In fact, suppose that $(a, b) \subset \mathbb{R}$ is an interval such that

$$
\begin{aligned}
& y(a) \in \partial B_{\epsilon / 2}(0), \quad y(b) \in \partial B_{\epsilon}(0) \\
& y(t) \in B_{\epsilon}(0) \backslash B_{\epsilon / 2}(0) \quad \text { in } t \in(a, b) .
\end{aligned}
$$

Then, we obtain

$$
a_{0} m_{\epsilon / 2}(b-a) \leq \int_{a}^{b}-a(t) W(y(t)) d t
$$

where $m_{\epsilon / 2}=\min _{x \in \mathbb{R}^{N} \backslash B_{\epsilon / 2}}(0)(-W(x))>0$. On account of Schwarz inequality, it follows that

$$
\begin{aligned}
\frac{\epsilon}{2} & \leq|y(b)-y(a)| \\
& \leq \int_{a}^{b}|\dot{y}(t)| d t \\
& \leq(b-a)^{1 / 2}\left(\int_{a}^{b}|\dot{y}(t)|^{2} d t\right)^{1 / 2} \\
& \leq \frac{1}{2} \int_{a}^{b}|\dot{y}(t)|^{2} d t+\frac{b-a}{2} \\
& \leq \frac{1}{2} \int_{a}^{b}|\dot{y}(t)|^{2} d t+\frac{1}{2 a_{0} m_{\epsilon / 2}} \int_{a}^{b}-a(t) W(y(t)) d t .
\end{aligned}
$$

If $y(t)$ intersects $\partial B_{\epsilon}(0)$ and $B_{\epsilon / 2}(0)$ infinitely as $t \rightarrow \infty$, we can find infinitely many disjoint intervals $\left(a_{i}, b_{i}\right)$ with the property (66). Thus, we have

$$
\frac{1}{2} \int_{\mathbb{R}}|\dot{y}(t)|^{2} d t+\frac{1}{2 a_{0} m_{\epsilon / 2}} \int_{\mathbb{R}}-a(t) W(y(t)) d t=\infty,
$$

which contradicts $\dot{y}(t) \in L^{2}\left(\mathbb{R}, \mathbb{R}^{N}\right)$ and (60). Therefore, we obtain $y(t) \rightarrow 0$ as $t \rightarrow \infty$.

Since $\widetilde{u}\left(t ; T_{k}\right)$ satisfies (HS), $\ddot{\widetilde{u}}\left(t ; T_{k}\right)=-a(t) W\left(\widetilde{u}\left(t ; T_{k}\right)\right)$ is bounded on each compact interval by (iii) and (61). Thus, $\widetilde{u}\left(t ; T_{k}\right)$ converges to $y(t)$ in $W_{\text {loc }}^{1, \infty}\left(\mathbb{R}, \mathbb{R}^{N}\right)$. Hence,

$$
\begin{aligned}
& \frac{1}{2}|\dot{y}(t)|^{2}+a(t) W(y(t)) \\
& =\lim _{k \rightarrow \infty}\left[\frac{1}{2}\left|\dot{\tilde{u}}\left(t ; T_{k}\right)\right|^{2}+a(t) W\left(\tilde{u}\left(t ; T_{k}\right)\right)\right] \\
& =\lim _{k \rightarrow \infty} E_{T_{k}}(t) \quad \forall t \in \mathbb{R} .
\end{aligned}
$$

Since $E_{T_{k}}(t)$ is increasing, $y(t) \rightarrow 0$ as $t \rightarrow \infty$ and $\dot{y}(t) \epsilon$ $L^{2}\left(\mathbb{R}, \mathbb{R}^{N}\right)$, it is obvious that $\dot{y}(t) \rightarrow 0$ as $t \rightarrow 0$.

Up to now, we are in the position to give the proof of our main result.

Proof. In view of Propositions 13 and 14, it is obvious that $y(t)$ is one nontrivial homoclinic solution of (HS).

\section{Conflict of Interests}

The authors declare that there is no conflict of interests regarding the publication of this paper.

\section{Acknowledgment}

The project is supported by the National Natural Science Foundation of China (Grant no. 11101304). 


\section{References}

[1] A. Ambrosetti and V. C. Zelati, Periodic Solutions of Singular Lagrangian Systems, vol. 10 of Progress in Nonlinear Differential Equations and Their Applications, Birkhäuser, Boston, Mass, USA, 1993.

[2] W. B. Gordon, "Conservative dynamical systems involving strong force," Transactions of the American Mathematical Society, vol. 204, pp. 113-115, 1975.

[3] P. H. Rabinowitz, "Periodic solutions of Hamiltonian systems," Communications on Pure and Applied Mathematics, vol. 31, no. 2, pp. 157-184, 1978.

[4] K. Tanaka, "Homoclinic orbits for a singular second order Hamiltonian system," Annales de l'Institut Henri Poincaré C, vol. 7, pp. 427-438, 1990.

[5] A. Bahri and P. H. Rabinowitz, "A minimax method for a class of Hamiltonian systems with singular potentials," Journal of Functional Analysis, vol. 82, no. 2, pp. 412-428, 1989.

[6] P. Caldiroli, "Existence and multiplicity of homoclinic orbits for potentials on unbounded domains," Proceedings of the Royal Society of Edinburgh A, vol. 124, no. 2, pp. 317-339, 1994.

[7] U. Bessi, "Multiple homoclinic orbits for autonomous singular potentials," Proceedings of the Royal Society of Edinburgh A, vol. 124, no. 4, pp. 785-802, 1994.

[8] J. Janczewska and J. Maksymiuk, "Homoclinic orbits for a class of singular second order Hamiltonian systems in $\mathbb{R}^{3}$," Central European Journal of Mathematics, vol. 10, no. 6, pp. 1920-1927, 2012.

[9] M. L. Bertotti and L. Jeanjean, "Multiplicity of homoclinic solutions for singular second-order conservative systems," Proceedings of the Royal Society of Edinburgh A, vol. 126, no. 6, pp. 1169-1180, 1996.

[10] P. Caldiroli and C. de Coster, "Multiple homoclinics for a class of singular Hamiltonian systems," Journal of Mathematical Analysis and Applications, vol. 211, no. 2, pp. 556-573, 1997.

[11] P. H. Rabinowitz, "Homoclinics for a singular Hamiltonian system," in Geometric Analysis and the Calculus of Variations, pp. 267-296, International Press, Cambridge, Mass, USA, 1996.

[12] P. Caldiroli and L. Jeanjean, "Homoclinics and heteroclinics for a class of conservative singular hamiltonian systems," Journal of Differential Equations, vol. 136, no. 1, pp. 76-114, 1997.

[13] M. J. Borges, "Heteroclinic and homoclinic solutions for a singular Hamiltonian system," European Journal of Applied Mathematics, vol. 17, no. 1, pp. 1-32, 2006.

[14] M. Izydorek and J. Janczewska, "The shadowing chain lemma for singular Hamiltonian systems involving strong forces," Central European Journal of Mathematics, vol. 10, no. 6, pp. 1928-1939, 2012.

[15] E. Séré, "Existence of infinitely many homoclinic orbits in Hamiltonian systems," Mathematische Zeitschrift, vol. 209, no. 1, pp. 27-42, 1992.

[16] E. Sere, "Looking for the Bernoulli shift," Annales de l'Institut Henri Poincaré C, vol. 10, pp. 561-590, 1993.

[17] P. Caldiroli and P. Montecchiari, "Homoclinic orbits for second order Hamiltonian systems with potential changing sign," Communications on Applied Nonlinear Analysis, vol. 1, pp. 97129, 1994.

[18] V. C. Zelati and P. H. Rabinowitz, "Homoclinic orbits for second order Hamiltonian systems possessing superquadratic potentials," Journal of the American Mathematical Society, vol. 4, no. 4, pp. 693-727, 1991.
[19] P. H. Rabinowitz, "Multibump solutions for an almost periodically forced singular Hamiltonian system," Electronic Journal of Differential Equations, vol. 1995, no. 12, pp. 1-21, 1995.

[20] M. Izydorek and J. Janczewska, "Connecting orbits for a periodically forced singular planar Newtonian system," Journal of Fixed Point Theory and Applications, vol. 12, no. 1-2, pp. 59-67, 2012.

[21] D. G. Costa and H. Tehrani, "On a class of singular secondorder Hamiltonian systems with infinitely many homoclinic solutions," Journal of Mathematical Analysis and Applications, vol. 412, no. 1, pp. 200-211, 2014.

[22] C. Greco, "Periodic solutions of a class of singular Hamiltonian systems," Nonlinear Analysis, vol. 12, no. 3, pp. 259-269, 1988.

[23] P. H. Rabinowitz, "Periodic and heteroclinic orbits for a periodic Hamiltonian system," Annales de l'Institut Henri Poincaré C, vol. 6, pp. 331-346, 1989. 


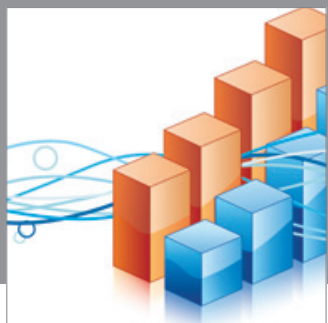

Advances in

Operations Research

mansans

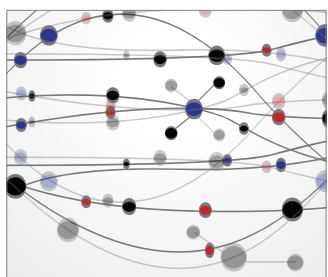

The Scientific World Journal
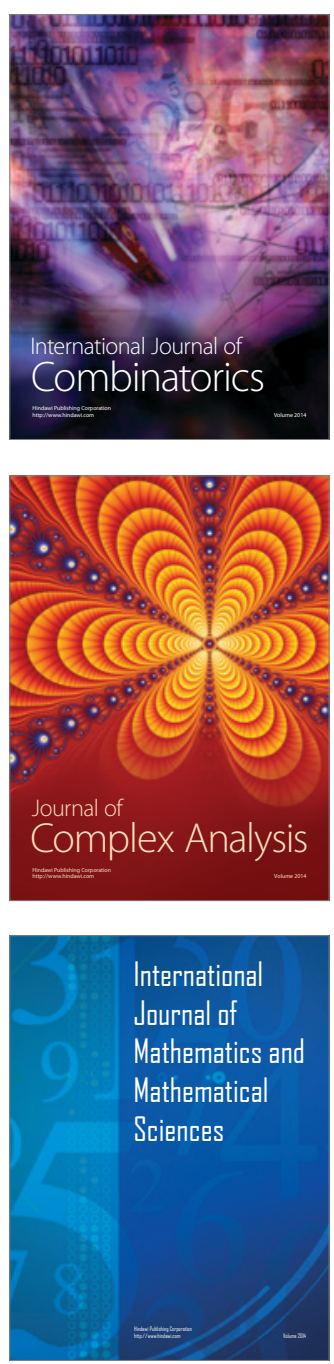
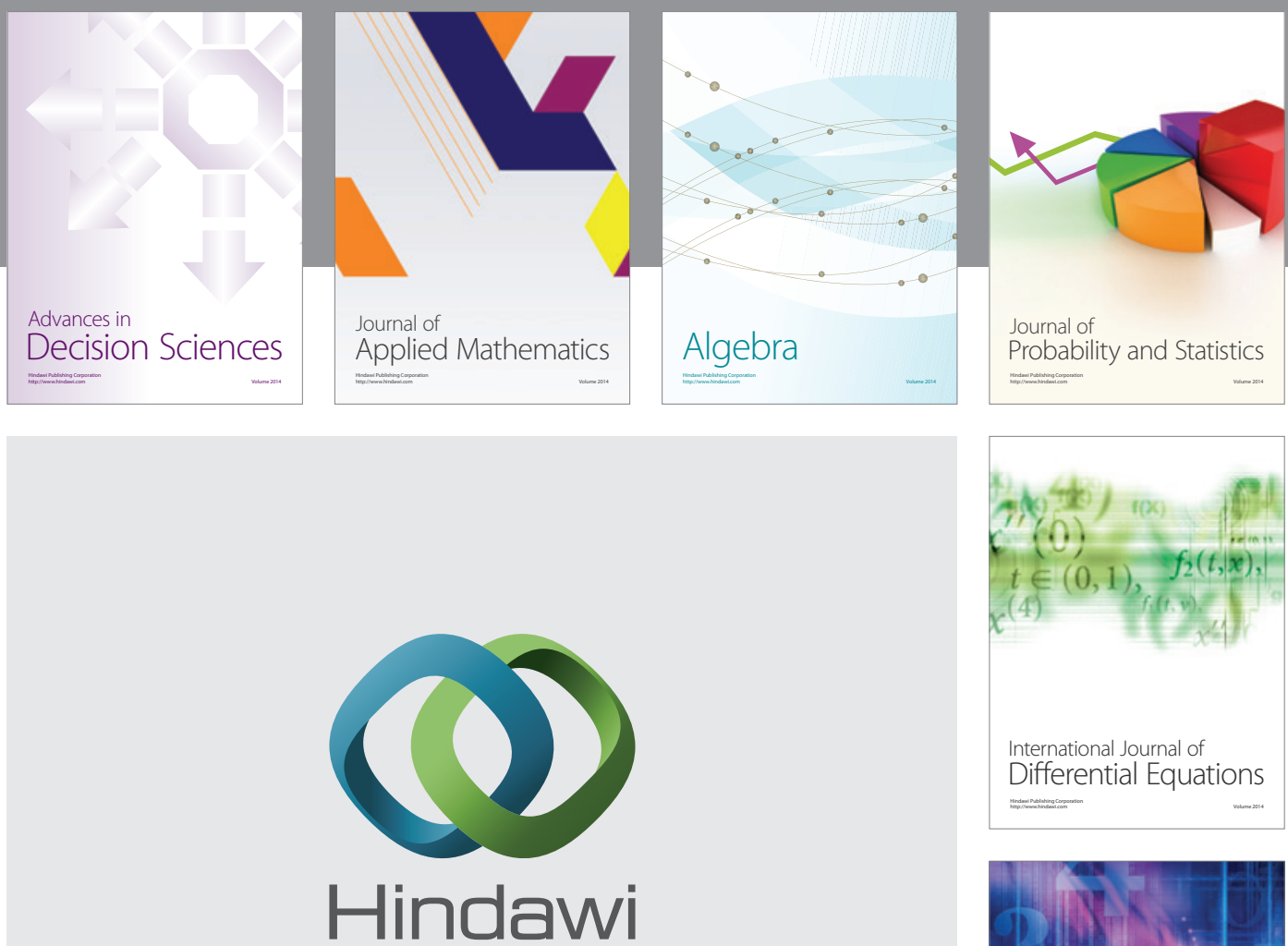

Submit your manuscripts at http://www.hindawi.com
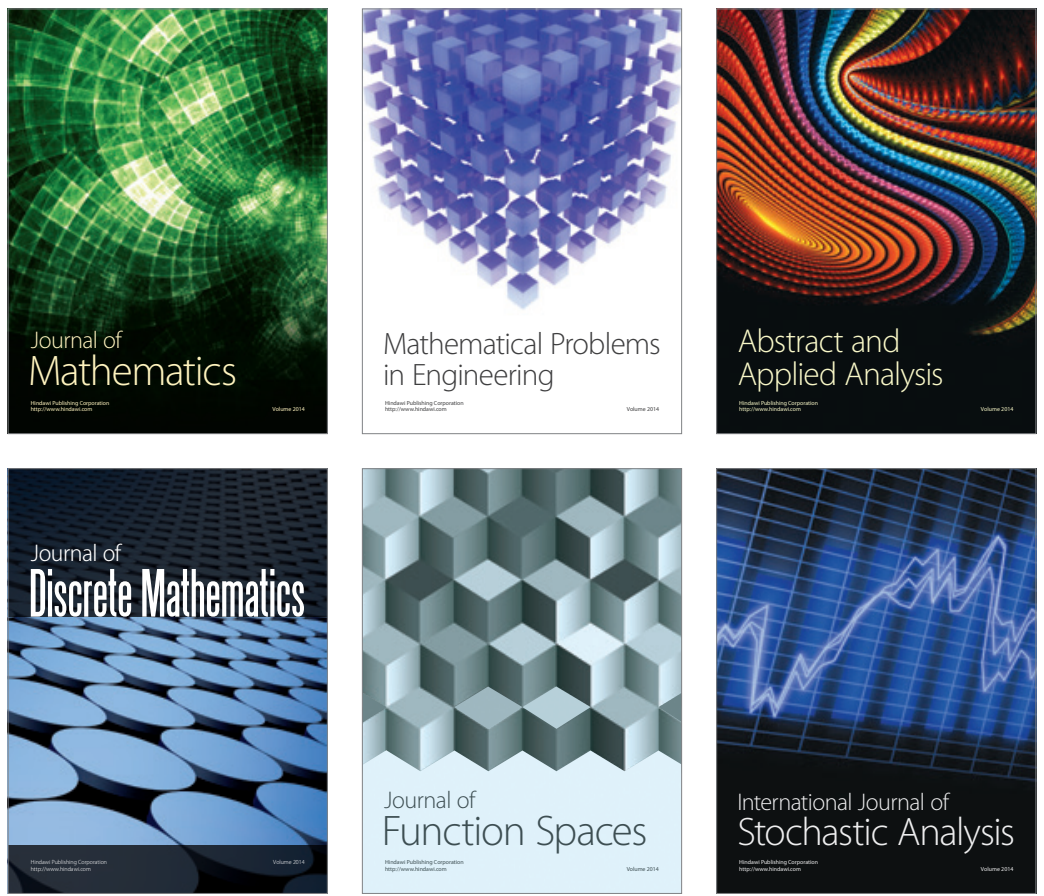

Journal of

Function Spaces

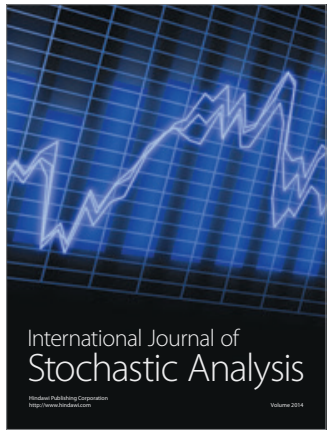

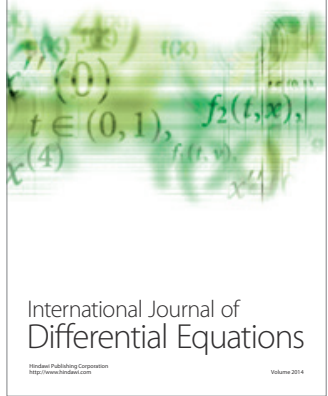
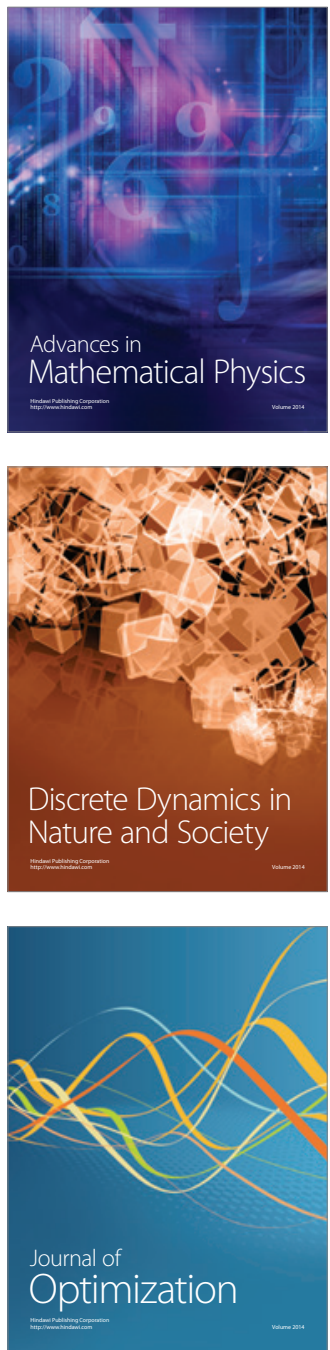